OPEN ACCESS

Edited by:

Roberto Esposito,

ASUR Marche, Italy

Reviewed by:

Su Xiaoyun,

Huazhong University of Science and Technology, China

Aleksandra Araszkiewicz,

Poznan University of Medical

Sciences, Poland

${ }^{*}$ Correspondence:

Felix T. Kurz

felix.kurz@med.uni-heidelberg.de

${ }^{\dagger}$ These authors share first authorship

Specialty section:

This article was submitted to

Brain Imaging Methods,

a section of the journal

Frontiers in Neuroscience

Received: 08 November 2021 Accepted: 17 December 2021

Published: 15 February 2022

Citation:

Jende JME, Mooshage $C$ Kender Z, Kopf S, Groener JB, Heiland S, Juerchott A, Nawroth $P$, Bendszus M and Kurz FT (2022) Magnetic Resonance Neurography

Reveals Smoking-Associated Decrease in Sciatic Nerve Structura

Integrity in Type 2 Diabetes.

Front. Neurosci. 15:811085.

doi: 10.3389/fnins.2021.811085

\section{Magnetic Resonance Neurography Reveals Smoking-Associated Decrease in Sciatic Nerve Structural Integrity in Type 2 Diabetes}

\author{
Johann M. E. Jende ${ }^{1 \dagger}$, Christoph Mooshage ${ }^{1 \dagger}$, Zoltan Kender ${ }^{2}$, Stefan Kopf ${ }^{2,3}$, \\ Jan B. Groener ${ }^{2,3}$, Sabine Heiland ${ }^{1,4}$, Alexander Juerchott' ${ }^{1}$, Peter Nawroth ${ }^{2,3,5}$, \\ Martin Bendszus ${ }^{1}$ and Felix T. Kurz ${ }^{1,6 *}$
}

\begin{abstract}
1 Department of Neuroradiology, Heidelberg University Hospital, Heidelberg, Germany, ${ }^{2}$ Department of Endocrinology, Diabetology and Clinical Chemistry, Heidelberg University Hospital, Heidelberg, Germany, ${ }^{3}$ German Center of Diabetes Research, München-Neuherberg, Germany, ${ }^{4}$ Division of Experimental Radiology, Department of Neuroradiology, Heidelberg, Germany, ${ }^{5}$ Joint Institute for Diabetes and Cancer at Helmholtz-Zentrum Munich and Heidelberg University, Heidelberg, Germany, ${ }^{6}$ Department of Radiology (E010), German Cancer Research Center, Heidelberg, Germany
\end{abstract}

Objective: It is controversially discussed in how far smoking contributes to diabetic polyneuropathy (DPN) in type 2 diabetes (T2D). Diffusion-weighted magnetic resonance neurography (MRN) at 3 Tesla has been shown to provide objective values for structural nerve integrity in patients with T2D. The aim of this study was to investigate the contribution of cigarette smoking on structural nerve integrity in T2D.

Methods: This cross-sectional prospective cohort study investigated the structural integrity of the sciatic nerve in 10 smokers, 40 never-smokers, and 20 ex-smokers with T2D and 10 healthy control subjects, using diffusion tensor imaging MRN at 3 Tesla and semi-automated nerve fiber tracking. Results were correlated with clinical, electrophysiological, and serological data.

Results: The sciatic nerve's fractional anisotropy (FA), a parameter for structural nerve integrity, was significantly lower in smokers with T2D when compared to controls $(p=0.002)$ and never-smokers $(p=0.015)$, and lower in ex-smokers when compared to controls $(p=0.015)$. In addition, sciatic nerve radial diffusivity, a marker of myelin damage, was increased in smokers versus controls and never-smokers $p=0.048$, $p=0.049$, respectively). Furthermore, FA in T2D patients was negatively correlated with clinical and electrophysiological markers of DPN. FA also showed negative correlations with the pulse wave velocity, a marker of arterial stiffness and associated microangiopathy, in controls $(r=-0.70 ; p=0.037)$, never-smokers $(r=-0.45$; $p=0.004)$, ex-smokers ( $r=-0.55 ; p=0.009)$, and a similar trend in smokers $(r=-0.63$; $p=0.076$ ). Negative correlations were found between FA and skin auto-fluorescence, a marker of tissue advanced glycation end product accumulation and therefore longterm glycemic stress in T2D, in never-smokers $(r=-0.39 ; p=0.020)$ and smokers $(r=-0.84 ; p=0.004)$, but not in ex-smokers $(r=-0.07 ; p=0.765)$. 
Conclusion: The findings indicate that smoking contributes to sciatic nerve damage in T2D, potentially worsening DPN due to glycemic stress and less microangiopathyassociated myelin damage in active smokers, while angiopathic effects predominate in ex-smokers. To stop smoking may therefore pose a promising preventive measure to slow the progression of DPN in T2D.

Keywords: smoking, diabetic polyneuropathy, magnetic resonance neurography (MRN), type 2 diabetes, diffusion tensor imaging

\section{INTRODUCTION}

Distal symmetric diabetic polyneuropathy (DPN) is one of the most disabling complications of diabetes with increasing prevalence affecting about 200 million patients worldwide causing high morbidity and extensive healthcare costs (Alleman et al., 2015). The poor effect of adjusting serum glucose on the course of DPN in type 2 diabetes (T2D) indicates that pathophysiological mechanisms other than the effects of hyperglycemia play an important role in the development of DPN (Tesfaye et al., 2005; Toth et al., 2012). Although several additional clinical and serological risk factors for developing DPN such as obesity, hypertension, hyperglycemia, dyslipidemia, and a decrease in renal function have been identified in clinical studies, the exact pathophysiological mechanisms underlying DPN remain poorly understood. As a result, there still is a lack of sufficient therapeutic strategies and preventive measures for this disorder (Gaede et al., 1999; Tesfaye et al., 2005; Elliott et al., 2009).

A broadly accepted hypothesis assumes that, in addition to glycation of surface molecules of axons and Schwann cells induced by hyperglycemia, nerve ischemia as a consequence of microangiopathy is another major contributor to nerve damage and the occurrence of painful symptoms in DPN, especially in T2D (Shillo et al., 2019). In this context, it has been discussed controversially whether cigarette smoking as a potential cause for microangiopathy contributes to damage of peripheral nerves in DPN (Benbow et al., 1997; Clair et al., 2015). Although several clinical studies concluded that smoking increases the risk for DPN, and it was found recently that smoking is associated with structural changes in dorsal root ganglia (Jende et al., 2020c), it has not yet been possible to quantify the amount and extent of structural nerve damage caused by smoking in vivo. Also, it remains to be determined whether the main contributing factor to nerve damage caused by smoking in T2D is either microangiopathy as a consequence of oxidative stress to the endothelium or oscillations of blood glucose induced by smoking (Csordas and Bernhard, 2013; Christensen et al., 2020).

It is further unknown whether damage to peripheral nerves caused by smoking is reversible once a patient stops smoking (Clair et al., 2015). Recent studies on high resolution magnetic resonance neurography (MRN) at three Tesla (3T) found that, in spite of the progression of clinical symptoms from distal to proximally, the maximum of visible nerve lesions occurs in the sciatic nerve at thigh level (Jende et al., 2020b). It

Abbreviations: DPN, diabetic polyneuropathy; T2D, type 2 diabetes; FA, fractional anisotropy; MRN, magnetic resonance neurography; NDS, Neuropathy Disability Score; NSS, Neuropathy Symptom Score. has further been demonstrated that parameters obtained from diffusion tensor imaging (DTI) can serve as reliable parameters for the assessment of structural nerve integrity in T2D (Heckel et al., 2015). In particular, previous studies found that the DTI fractional anisotropy (FA) of peripheral nerves was positively correlated with electrophysiological parameters of both axonal and myelin sheath integrity, whereas axial diffusivity showed positive correlations with parameters of axonal integrity and radial diffusivity showed negative correlations with parameters of myelin sheath integrity (Jende et al., 2020a).

The aim of this study was to investigate the impact of smoking on the sciatic nerve's structural integrity in patients with T2D by combining diffusion-weighted 3T MRN of the sciatic nerve with detailed clinical, serological and electrophysiological parameters obtained from smokers, ex-smokers and never-smokers with T2D as well as healthy controls.

\section{MATERIALS AND METHODS}

\section{Study Design and Participants}

This study was approved by the local ethics committee (clinicaltrials.gov identifier NCT03022721) and all participants gave written informed consent. Participants were screened and recruited at the outpatient clinic of the Department of Endocrinology at Heidelberg University Hospital, where all clinical, serological, and electrophysiological examinations took place. Thereafter, patients underwent MRN at the Department of Neuroradiology at Heidelberg University Hospital, where image processing was performed. All patient data were pseudonymized and participating researchers at the Department of Neuroradiology were completely blinded to all patient data. In total, 70 patients with T2D (10 active smokers, 20 exsmokers, 40 never-smokers; female: 31; male: 39) and 10 age-matched controls ( 7 women, 3 men) took part in this single-center study between September 2016 and June 2020. Patients with T2D were divided into three groups (smokers, exsmokers, and never-smokers). Smokers were identified first, and, thereafter, never-smokers and ex-smokers were matched for age, sex, body-mass index (BMI), hemoglobin A1c (HbA1c) levels, cholesterol levels, parameters of renal function, medication, and disease duration, in order to minimize confounding factors that might influence structural nerve integrity. Matching was accepted as adequate when no significant difference was found for each pair-wise group comparison. Recruitment proved to be time-consuming due to difficulties in enrolling smokers with T2D to undergo the extensive study protocol, the subsequent detailed matching process of ex-smokers, 
never-smokers, and healthy participants, as well as the COVID19 pandemic.

The control group was matched to the three groups for age, BMI, lipid profile, and parameters of renal function. Detailed information on patient recruitment is given in Figure 1.

For all participants, exclusion criteria were age $<18$, pregnancy, any contraindications for MR imaging, any history of lumbar surgery or disk protrusion, any other risk factors for neuropathy such as alcoholism, malignant or infectious diseases, hypovitaminosis, monoclonal gammopathy, any previous or ongoing exposure to neurotoxic agents, and any chronic neurological diseases such as Parkinson's disease, restless leg syndrome, or multiple sclerosis. To exclude severe renal insufficiency as potential confounders, only patients with an estimated glomerular filtration rate (eGFR) $>60 \mathrm{ml} / \mathrm{min}$ were included in this study.

\section{Clinical and Electrophysiological Examination}

For every patient, a detailed medical history was documented. Patients with T2D who had never smoked were assigned to the group of never-smokers, patients who had stopped smoking $>1$ year ago were assigned to the group of ex-smokers and patients who were actively smoking were assigned to the group of smokers. Control subjects who had never smoked were assigned to the control group.

An examination of neuropathic symptoms was performed according to the guidelines issued by the German Society for Diabetology, including evaluation of the neuropathy disability score (NDS) and the neuropathy symptom score (NSS) (Young et al., 1993).

The electrophysiological examination (Viasys Healthcare VikingQuest ${ }^{\circledR}$, Viasys Healthcare $\mathrm{GmbH}$, Höchberg) of the right leg included: distal motor latencies (DML) of the right tibial and peroneal nerve, motor and sensory amplitudes [compound muscle action potentials (CMAPs), and sensory nerve action potentials (SNAPs), respectively] of the tibial, peroneal, and sural nerves, and nerve conduction velocities (NCVs) of the tibial, peroneal, and sural nerves. All examinations were performed in accordance with international standards for electrophysiological examinations and with specifications as issued by the manufacturer of the testing device, see also Stålberg et al. (2019) and Natus Medical Incorporated (2020) for further details. It was assured that skin temperature was at least $32^{\circ} \mathrm{C}$ throughout the examination.

The intima media thickness (IMT) was assessed with duplex ultrasonographic examination of both carotid arteries (SonoAce X8; Samsung Group). The pulse wave velocity was calculated using non-invasive blood pressure measurements of the arms and ankles (ABI System 1000; Boso d.o.o.).

Dermal accumulation of advanced glycation end products (AGE) was assessed non-invasively measuring skin auto fluorescence (SAF) with an AGE reader (AGE Reader SU, DiagnOptics BV, Netherlands). A skin surface of about $4 \mathrm{~cm}^{2}$ was assessed at the volar surface of the forearm (Fernando et al., 2019). For the calculation of SAF, the average emitted light intensity per $\mathrm{nm}$ was divided by the average extinction light intensity per nm and multiplied by 100 . SAF is expressed in arbitrary units (AU) (Corine van de Zande et al., 2020). Blood was drawn in fasting state and processed immediately under standardized conditions in the central laboratory of Heidelberg University Hospital. Albumin excretion in urine was detected in morning spot urine within all participants. Estimated glomerular filtration rate was calculated with the CKD-EPI-formula (Levey et al., 2009).

\section{Magnetic Resonance Neurography Imaging Protocol}

All participants underwent high-resolution MRN of the right leg in a 3.0 Tesla MR-scanner (Magnetom TIM-TRIO, Siemens, Erlangen, Germany). A 15-channel transmit-receive extremity coil was used, and the following sequence protocol was applied:

(1) an axial high resolution T2-weighted turbo spin echo 2D sequence with spectral fat saturation of the right mid-thigh and the following parameters: repetition time (TR) $5970 \mathrm{~ms}$, echo time (TE) $55 \mathrm{~ms}$, field of view (FOV) $160 \times 160 \mathrm{~mm}^{2}$, matrix size $512 \times 512$, slice thickness $4 \mathrm{~mm}$, interslice gap $=0.35 \mathrm{~mm}$, voxel size $0.3 \times 0.3 \times 4.0 \mathrm{~mm}^{3}, 3$ averages, 24 images.

(2) DTI with an axial fat-suppressed, diffusion-weighted two-dimensional echo-planar sequence with the following parameters: $\mathrm{TR}=5100 \mathrm{~ms} ; \mathrm{TE}=92.8 \mathrm{~ms} ; \mathrm{b}=0$ and $1000 \mathrm{~s} / \mathrm{mm}^{2}$; directions $=20 ; \mathrm{FOV}=160 \times 160 \mathrm{~mm}^{2} ;$ matrix size $=128 \times 128$; slice thickness $=4 \mathrm{~mm}$; voxel size $=1.3 \times 1.3 \times 4 \mathrm{~mm}^{3}$; no interslice gap, 3 averages, 24 slices, 1512 images.

\section{Image Post-processing}

All images were pseudonymized and subsequently analyzed in an automated approach using Nordic BRAINEX (NordicNeuroLab AS, 2019), a United States Food and Drug Administrationapproved processing software designed for automated calculation and reconstruction of fiber tracts in diffusion-weighted imaging (Christidi et al., 2016). A total of $84 \times 1536=129,024$ images were analyzed accordingly. T2-weighted and diffusion-weighted sequences were co-registered, and the region of the sciatic nerve was marked by two trained neuroradiologists with 6 and 2 years of experience in MRN imaging, respectively. The nerve was automatically segmented with a threshold of $>0.1$ for the nerve's FA, a dimensionless quantity that measures directed diffusion, with values between 0 (isotropic diffusion) and 1 (diffusion in only one direction). A tract turning angle of 41.4 degrees, a minimum fiber length of $20 \mathrm{~mm}$, and one seed per voxel were chosen, as done in previous studies (Jende et al., 2021). After nerve fibers were tracked, the nerve tensor eigenvalues $\lambda 1, \lambda 2$, and $\lambda 3$ and the average FA were automatically determined by Nordic BRAINEX. Axial diffusivity (AD), radial diffusivity (RD), and mean diffusivity (MD) were subsequently calculated based on the obtained tensor eigenvalues as $\mathrm{AD}=\lambda 1$, $\mathrm{RD}=(\lambda 2+\lambda 3) / 2$, and $\mathrm{MD}=(\lambda 1+\lambda 2+\lambda 3) / 3$. An illustration of the process of automated nerve segmentation is given in Figure 2.

\section{Statistical Analysis}

Statistical data analysis was performed with GraphPad Prism 6. All data were tested for Gaussian normal distribution using 


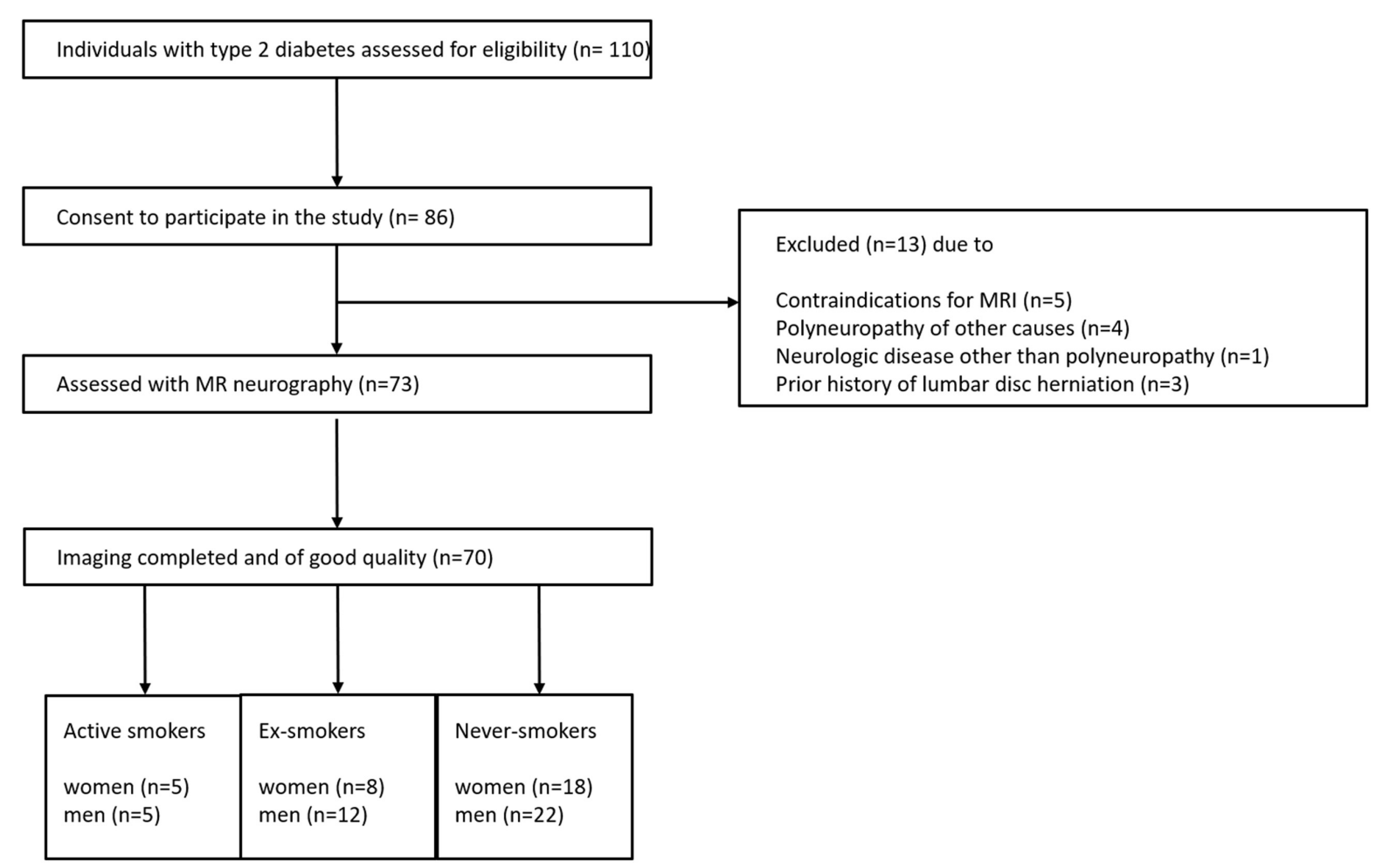

FIGURE 1 | Patient recruitment flowchart.

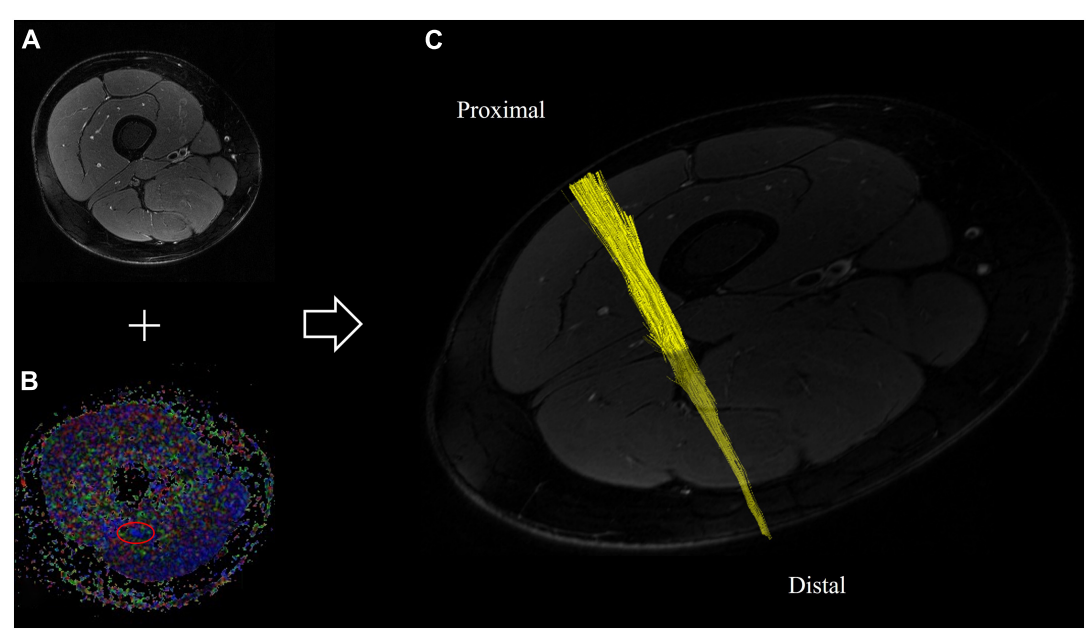

FIGURE 2 | Automated segmentation of the sciatic nerve based on diffusion-weighted and T2-weighted imaging. (A) T2-weighted cross-sectional image of the right thigh, and (B) corresponding fractional anisotropy map. The sciatic nerve is delineated in red. (C) Reconstructed fiber tracts of the sciatic nerve based on diffusion-tensor imaging of the proximal thigh.

the D'Agostino-Pearson omnibus normality test. If a Gaussian normal distribution was given, $t$-tests were used for comparisons of two groups, one-way ANOVAs with Tukey correction for multiple comparisons were applied for comparisons of more than two groups and Bonferroni-corrected Pearson correlation coefficients were calculated for correlation analysis. If data were not Gaussian distributed, the Mann-Whitney test was used for comparisons of two groups, the Kruskal-Wallis test with Dunn correction for multiple comparisons was used for multiple comparisons of more than three groups and non-parametric Spearman correlations were applied for correlation analysis. For all tests, the level of significance was defined at $p<0.05$. 


\section{RESULTS}

\section{Clinical and Epidemiological Data}

Ten active smokers (5 women, 5 men, 15.79 pack years \pm 14.19 ; range: $1-57$, mean age $53.20 \pm 12.85$ years, BMI $30.48 \pm 3.60 \mathrm{~kg} / \mathrm{m}^{2}$, disease duration $10.60 \pm 12.30$ years) with T2D, 20 ex-smokers ( 8 women, 12 men; $20.09 \pm 12.05$ years after smoking, mean age $59.42 \pm 6.87$ years, BMI $28.42 \pm 5.52 \mathrm{~kg} / \mathrm{m}^{2}$, disease duration $11.79 \pm 10.54$ years) with T2D, 40 neversmokers (18 women, 22 men, mean age $57.44 \pm 14.01$ years, BMI $28.45 \pm 5.02 \mathrm{~kg} / \mathrm{m}^{2}$, disease duration $12.93 \pm 11.21$ years) with T2D, and 10 age matched controls ( 7 women, 3 men, mean age $49.80 \pm 12.89$ years, BMI $28.05 \pm 5.69 \mathrm{~kg} / \mathrm{m}^{2}$ ) took part in this study. For all groups, ANOVA showed no differences for age $(p=0.186)$, sex $(p=0.151)$, BMI $(p=0.678)$ or NDS scores $(p=0.103)$.

For T2D patient groups, no significant difference in disease duration was found $(p=0.744)$. Oral antidiabetic medication (metformin) was used by 4 smokers, 8 ex-smokers, and 13 neversmokers. Insulin was used by 4 smokers, 5 ex-smokers, and 11 never smokers. A total of 42 participants with T2D suffered from hypertension. Anti-hypertensive medication (beta blockers, renin-angiotensin-aldosterone inhibitors, calcium antagonists) were used by 8 smokers, 12 ex-smokers, and 22 never-smokers. Diuretics (thiazides, loop diuretics) were taken by 5 smokers, 4 ex-smokers, and 6 never-smokers. $100 \mathrm{mg}$ aspirin daily was taken by 2 active smokers, 7 ex-smokers, and 7 never-smokers. Statins were taken by 5 active smokers, 10 ex-smokers, and 16 never-smokers.

Compared to controls, NSS scores were higher in ex-smokers ( $p=0.026)$ and never-smokers $(p=0.036)$, but not in smokers $(p=0.233)$. There was no difference between the NSS scores of T2D patient groups. The pulse wave velocity (PWV), a marker of arterial stiffness and associated microangiopathy (Kim and Kim, 2019), was higher in ex-smokers compared to controls $(p=0.036)$.

\section{Serological Data}

HbA1c levels were higher in never-smokers and ex-smokers when compared to controls $(p=0.003$ and $p=0.005$, respectively). There was no significant difference between $\mathrm{HbAlc}$ levels of T2D patient groups. High sensitivity Troponin T (hsTNT), a marker of cardiovascular disease, was higher in ex-smokers when compared to controls $(p=0.038)$. Highdensity lipoprotein (HDL) cholesterol was higher in smokers in comparison to ex-smokers $(p=0.016)$. No significant differences were found for cystatin $c$, glomerular filtration rate (GFR), N-terminal pro-brain natriuretic peptide (proBNP), total serum cholesterol, low-density lipoprotein (LDL) cholesterol, or triglycerides.

\section{Electrophysiological Data}

Tibial NCV was higher in controls compared to ex-smokers and smokers ( $p=0.047$ and $p=0.018$, respectively). Peroneal NCV was higher in controls compared to ex-smokers $(p=0.012)$ and to smokers ( $p=0.033$ ). No significant differences were found for sural NCV and amplitudes, tibial and peroneal CMAP, or tibial and peroneal DML. A detailed summary of all group comparisons on epidemiological, electrophysiological, and serological data is provided in Table 1.

\section{Magnetic Resonance Neurography Imaging Data \\ Comparison of Groups and Correlation With Clinical Scores and Demographic Data}

FA as the main imaging parameter for structural nerve integrity, was higher in controls when compared to ex-smokers $(p=0.015)$ and smokers $(p=0.002)$ but not when compared to neversmokers $(p=0.361$; Figure 3A). FA was also higher in neversmokers when compared to smokers $(p=0.015)$. A summary of all group comparisons for imaging data is provided in Table $\mathbf{1}$. In controls, FA showed negative correlations with age $(r=-0.75$; $p=0.013)$, likewise in never smokers $(r=-0.50 ; p=0.001)$. This finding could not be reproduced for ex-smokers and smokers. In smokers with $\mathrm{T} 2 \mathrm{D}, \mathrm{FA}$ showed negative correlations with BMI $(r=-0.75 ; p=0.013)$. In never-smokers, ex-smokers and smokers with T2D, FA was negatively correlated with the NDS score $(r=-0.49 ; p=0.001, r=-0.52 ; p=0.031$, and $r=-0.70 ; p=0.024$, respectively). In smokers, FA also showed a negative correlation with the NSS score $(r=-0.69, p=0.028)$. No correlations were found with pack years or the average number of cigarettes per day in smokers. A detailed survey of correlations between FA and all other parameters acquired is provided in Table 2.

For $\mathrm{RD}$, a parameter indicative for the damage to myelin, ANOVA showed higher values in smokers compared to controls ( $p=0.048)$ and never-smokers ( $p=0.049$; Figure 3B).

In never-smokers, $\mathrm{RD}$ was correlated with age $(r=0.44$; $p=0.005)$. In smokers, RD was correlated with the BMI ( $r=0.68$; $p=0.042)$. RD was positively correlated with the NDS score in never-smokers $(r=0.39 ; p=0.017)$ and smokers $(r=0.68$; $p=0.046)$, but not in ex-smokers $(r=0.275 ; p=0.286)$. No correlations were found with the NSS score. A detailed survey of correlations between $\mathrm{RD}$ and all other parameters is provided in Table 3.

For all groups, no significant differences were found for $\mathrm{AD}$, a parameter supposed to represent axonal integrity (Figure 3C). In smokers, $\mathrm{AD}$ was correlated with the BMI $(r=0.76$; $p=0.011)$. No other correlations were found for $\mathrm{AD}$ with any of the acquired clinical scores or demographic data. A detailed survey of correlations of $\mathrm{AD}$ and with all other parameters is provided in Table 4.

\section{Correlation With Electrophysiological Data}

FA showed positive correlations with tibial and peroneal NCV in never-smokers $(r=0.34 ; p=0.042$ and $r=0.41 ; p=0.014$, respectively), ex-smokers ( $r=0.60 ; p=0.007$, and $r=0.70$; $p<0.001$, respectively), and smokers $(r=0.67 ; p=0.034$ and $r=0.85 ; p=0.003$, respectively) with T2D. Further positive correlations were found between FA and tibial and peroneal CMAP in never-smokers $(r=0.45 ; p=0.006$ and $r=0.58$; $p<0.001$, respectively) and ex-smokers $(r=0.55 ; p=0.014$ and $r=0.64 ; p=0.003$, respectively), while a similar trend was found in smokers $(r=0.51 p=0.136$ and $r=0.64$; $p=0.066$, respectively). 
TABLE 1 | Comparison of imaging, clinical, electrophysiological, apparatus-bound, and serologic parameters between controls, type 2 diabetes (T2D) never-smokers, ex-smokers, and smokers.

\begin{tabular}{|c|c|c|c|c|c|c|c|c|c|c|c|}
\hline Parameter & Controls & T2D never-smokers & T2D ex-smokers & T2D smokers & $P$-Value & $\begin{array}{c}P \text {-Value } \\
\text { controls } \\
\text { vs. never- } \\
\text { smokers }\end{array}$ & $\begin{array}{l}P \text {-Value } \\
\text { controls } \\
\text { vs. ex- } \\
\text { smokers }\end{array}$ & $\begin{array}{c}P \text {-Value } \\
\text { controls } \\
\text { vs. } \\
\text { smokers }\end{array}$ & $\begin{array}{c}\text { P-Value } \\
\text { never- } \\
\text { smokers vs. } \\
\text { ex-smokers }\end{array}$ & $\begin{array}{c}P \text {-Value } \\
\text { never- } \\
\text { smokers vs. } \\
\text { smokers }\end{array}$ & $\begin{array}{c}P \text {-Value } \\
\text { ex-smokers } \\
\text { vs. smokers }\end{array}$ \\
\hline $\begin{array}{l}\text { Fractional } \\
\text { anisotropy }\end{array}$ & $0.52 \pm 0.04$ & $0.48 \pm 0.06$ & $0.43 \pm 0.09$ & $0.40 \pm 0.08$ & $<0.001$ & 0.361 & 0.015 & 0.002 & 0.126 & 0.015 & 0.601 \\
\hline $\begin{array}{l}\text { Radial diffusivity } \\
{\left[10^{-5} \mathrm{~mm}^{2} / \mathrm{s}\right]}\end{array}$ & $73.78 \pm 11.94$ & $77.71 \pm 10.42$ & $88.33 \pm 24.44$ & $93.53 \pm 18.82$ & 0.008 & 0.904 & 0.105 & 0.048 & 0.091 & 0.049 & 0.856 \\
\hline $\begin{array}{l}\text { Axial diffusivity } \\
{\left[10^{-5} \mathrm{~mm}^{2} / \mathrm{s}\right]}\end{array}$ & $182.80 \pm 23.58$ & $171.80 \pm 19.41$ & $172.80 \pm 30.86$ & $174.40 \pm 14.05$ & 0.592 & 0.523 & 0.676 & 0.843 & 0.999 & 0.988 & 0.998 \\
\hline Women/Men & $7 w 3 m$ & 18w22m & 8w12m & $5 w 5 m$ & 0.151 & 0.170 & 0.124 & 0.531 & 0.961 & 0.983 & 0.904 \\
\hline Age (years) & $49.80 \pm 12.89$ & $57.44 \pm 14.01$ & $59.42 \pm 6.87$ & $53.20 \pm 12.85$ & 0.186 & 0.309 & 0.200 & 0.927 & 0.939 & 0.768 & 0.573 \\
\hline $\begin{array}{l}\text { Body-mass index } \\
\left(\mathrm{kg} / \mathrm{m}^{2}\right)\end{array}$ & $28.05 \pm 5.69$ & $28.45 \pm 5.02$ & $28.42 \pm 5.52$ & $30.48 \pm 3.6$ & 0.508 & 0.996 & 0.997 & 0.712 & $>0.999$ & 0.677 & 0.730 \\
\hline NDS & $0.30 \pm 0.67$ & $2.28 \pm 2.85$ & $2.94 \pm 3.27$ & $2.70 \pm 2.67$ & 0.103 & 0.22 & 0.097 & 0.22 & 0.777 & 0.892 & 0.892 \\
\hline NSS & $0 \pm 0$ & $3.31 \pm 3.21$ & $3.78 \pm 3.70$ & $3.10 \pm 3.41$ & 0.027 & 0.036 & 0.026 & 0.232 & $>0.999$ & $>0.999$ & $>0.999$ \\
\hline Tibial NCV (m/s) & $47.10 \pm 7.75$ & $42.65 \pm 5.07$ & $40.76 \pm 5.31$ & $38.90 \pm 8.01$ & 0.017 & 0.151 & 0.047 & 0.018 & 0.488 & 0.227 & 0.489 \\
\hline Tibial CMAP $(\mu \bigvee)$ & $15.56 \pm 8.18$ & $12.91 \pm 9.43$ & $1212 \pm 6.68$ & $9.45 \pm 5.91$ & 0.427 & 0.808 & 0.728 & 0.361 & 0.988 & 0.648 & 0.851 \\
\hline Tibial DML (ms) & $5.39 \pm 3.18$ & $7.46 \pm 4.89$ & $5.51 \pm 3.63$ & $6.53 \pm 5.72$ & 0.398 & 0.583 & $>0.999$ & 0.944 & 0.465 & 0.940 & 0.943 \\
\hline Peroneal NCV (m/s) & $46.00 \pm 3.30$ & $42.14 \pm 6.17$ & $39.24 \pm 5.12$ & $39.11 \pm 6.60$ & 0.008 & 0.247 & 0.012 & 0.033 & 0.525 & 0.852 & $>0.999$ \\
\hline $\begin{array}{l}\text { Peroneal CMAP } \\
(\mu \mathrm{V})\end{array}$ & $7.81 \pm 2.08$ & $5.83 \pm 3.40$ & $4.99 \pm 2.79$ & $4.54 \pm 4.49$ & 0.069 & 0.519 & 0.214 & 0.076 & $>0.999$ & 0.900 & $>0.999$ \\
\hline Peroneal DML (ms) & $4.45 \pm 1.81$ & $6.43 \pm 3.84$ & $5.49 \pm 2.87$ & $6.67 \pm 4.55$ & 0.401 & 0.433 & 0.901 & 0.550 & 0.800 & 0.998 & 0.849 \\
\hline Sural NCV (m/s) & $48.78 \pm 7.24$ & $46.17 \pm 6.00$ & $45.92 \pm 5.58$ & $45.05 \pm 9.94$ & 0.693 & $>0.999$ & $>0.999$ & $>0.999$ & $>0.999$ & $>0.999$ & $>0.999$ \\
\hline Sural SNAP $(\mu V)$ & $9.78 \pm 6.82$ & $7.44 \pm 4.49$ & $5.16 \pm 3.21$ & $4.95 \pm 3.48$ & 0.054 & 0.515 & 0.077 & 0.134 & 0.348 & 0.501 & $>0.999$ \\
\hline $\operatorname{SAF}(A U)$ & $1.81 \pm 0.37$ & $2.15 \pm 0.54$ & $2.28 \pm 0.48$ & $2.32 \pm 0.53$ & 0.072 & 0.344 & 0.091 & 0.162 & $>0.999$ & $>0.999$ & $>0.999$ \\
\hline $\begin{array}{l}\text { Pulse wave velocity } \\
(\mathrm{m} / \mathrm{s})\end{array}$ & $7.30 \pm 1.56$ & $8.26 \pm 1.65$ & $9.32 \pm 1.78$ & $8.75 \pm 1.92$ & 0.036 & 0.355 & 0.036 & 0.249 & 0.187 & 0.654 & 0.654 \\
\hline $\begin{array}{l}\text { Intima-media } \\
\text { thickness (ratio) }\end{array}$ & $0.97 \pm 0.21$ & $0.92 \pm 0.25$ & $0.96 \pm 0.23$ & $0.75 \pm 0.23$ & 0.114 & 0.915 & 0.999 & 0.172 & 0.914 & 0.194 & 0.116 \\
\hline $\mathrm{HbA1c}(\%)$ & $5.45 \pm 0.17$ & $7.28 \pm 1.90$ & $7.05 \pm 1.02$ & $6.33 \pm 1.29$ & 0.002 & 0.003 & 0.005 & 0.467 & $>0.999$ & 0.852 & 0.802 \\
\hline Cystatin C (mg/l) & $0.73 \pm 0.11$ & $0.82 \pm 0.17$ & $0.9 \pm 0.21$ & $0.91 \pm 0.26$ & 0.185 & 0.568 & 0.173 & 0.177 & 0.551 & 0.543 & 0.996 \\
\hline $\begin{array}{l}\text { Glomerular filtration } \\
\text { rate (ml/min) }\end{array}$ & $92.64 \pm 8.97$ & $88.39 \pm 16.75$ & $89.59 \pm 17.90$ & $79.36 \pm 20.93$ & 0.386 & 0.922 & 0.974 & 0.381 & 0.996 & 0.505 & 0.468 \\
\hline $\begin{array}{l}\text { Total serum } \\
\text { cholesterol (mg/dl) }\end{array}$ & $204.80 \pm 48.38$ & $196.20 \pm 41.63$ & $189.60 \pm 46.23$ & $195.20 \pm 45.71$ & 0.865 & 0.958 & 0.854 & 0.968 & 0.956 & $>0.999$ & 0.989 \\
\hline $\begin{array}{l}\text { HDL cholesterol } \\
\text { (mg/dl) }\end{array}$ & $54.63 \pm 12.06$ & $55.15 \pm 16.55$ & $45.59 \pm 10.64$ & $67.80 \pm 22.29$ & 0.077 & $>0.999$ & 0.614 & $>0.999$ & 0.190 & 0.632 & 0.016 \\
\hline $\begin{array}{l}\text { LDL cholesterol } \\
(\mathrm{mg} / \mathrm{dl})\end{array}$ & $131.00 \pm 39.60$ & $110.90 \pm 35.44$ & $110.30 \pm 39.37$ & $107.60 \pm 41.03$ & 0.475 & 0.861 & $>0.999$ & 0.879 & $>0.999$ & $>0.999$ & $>0.999$ \\
\hline Triglycerides (mg/dl) & $78.25 \pm 15.35$ & $79.95 \pm 28.37$ & $68.12 \pm 14.69$ & $77.90 \pm 32.58$ & 0.462 & 0.999 & 0.789 & $>0.999$ & 0.412 & 0.998 & 0.770 \\
\hline Troponin T (pg/dl) & $8.24 \pm 8.69$ & $7.93 \pm 3.29$ & $12.81 \pm 7.62$ & $10.90 \pm 6.76$ & 0.009 & $>0.999$ & 0.038 & 0.530 & 0.066 & $>0.999$ & $>0.999$ \\
\hline proBNP (pg/dl) & $24.13 \pm 9.48$ & $25.05 \pm 9.08$ & $27.00 \pm 9.16$ & $21.90 \pm 8.48$ & 0.554 & 0.994 & 0.881 & 0.955 & 0.882 & 0.762 & 0.496 \\
\hline
\end{tabular}

All values are displayed as mean \pm standard deviation.

NDS, Neuropathy Disability Score; NSS, Neuropathy Symptom Score; NCV, Nerve Conduction Velocitie; CMAP, compound muscle action potential; DML, Peroneal Distal Motor Latencies; SNAP, Sensory Nerve Action Potential; SAF, Skin Auto Fluorescence; HbA1c, hemoglobin A1c; HDL, High-Density Lipoprotein; LDL, Low-Density Lipoprotein; proBNP, pro-Brain Natriuretic Peptide; m/s, meters per second; mm, millimeter, $\mu$ V, microvolt; ms, milliseconds; AU, arbitrary units; mg/dl, milligram per deciliter; mg/l, milligram per liter; ml/min, milliliters per minute; pg/dl, picogram per deciliter. 

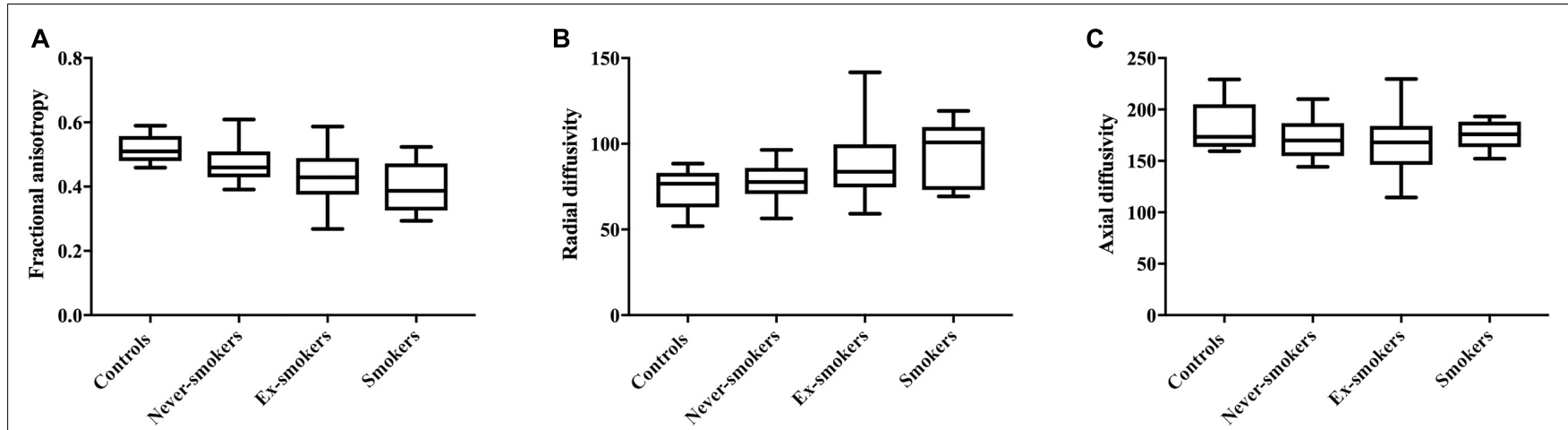

FIGURE 3 | Parameters derived from diffusion weighted MRN in all groups. (A) Fractional anisotropy in controls (0.52 \pm 0.04$)$, never-smokers (0.48 \pm 0.06$)$, ex-smokers (0.43 \pm 0.09$)$, and smokers $(0.40 \pm 0.08) ; F=6.324: r^{2}=0.200 ; p<0.001$. (B) Radial diffusivity in controls (73.78 \pm 11.94$)$, never-smokers (77.71 \pm 10.42$)$, ex-smokers (88.33 \pm 24.44$)$, and smokers (93.53 \pm 18.82$) ; F=4.264 ; r^{2}=0.147 ; p=0.008$. (C) Axial diffusivity in controls (182.80 \pm 23.58$)$, never-smokers (171.80 \pm 19.41$)$, ex-smokers (172.80 \pm 30.86$)$, and smokers $(174.40 \pm 14.05) ; F=1.845 ; r^{2}=0.026 ; p=0.570$.

TABLE 2 | Correlation of the sciatic nerve's fractional anisotropy (FA) with demographic, clinical, apparatus-bound, and serological parameters.

\begin{tabular}{|c|c|c|c|c|c|c|c|c|}
\hline & \multicolumn{2}{|c|}{ FA controls } & \multicolumn{2}{|c|}{ FA T2D never-smokers } & \multicolumn{2}{|c|}{ FA T2D ex-smokers } & \multicolumn{2}{|c|}{ FA T2D smokers } \\
\hline & $r$ & $p$ & $r$ & $p$ & $r$ & $p$ & $r$ & $p$ \\
\hline Radial diffusivity & -0.26 & 0.469 & -0.67 & $<0.001$ & -0.89 & $<0.001$ & -0.80 & 0.010 \\
\hline Axial diffusivity & 0.36 & 0.305 & 0.29 & 0.071 & -0.35 & 0.144 & -0.65 & 0.044 \\
\hline Sex & 0.01 & 0.974 & 0.16 & 0.328 & 0.22 & 0.347 & 0.29 & 0.410 \\
\hline Age (years) & -0.75 & 0.013 & -0.50 & 0.001 & -0.21 & 0.372 & -0.47 & 0.173 \\
\hline Body-mass index $\left(\mathrm{kg} / \mathrm{m}^{2}\right)$ & 0.17 & 0.635 & -0.29 & 0.074 & -0.003 & 0.990 & -0.75 & 0.013 \\
\hline NDS & -0.02 & 0.955 & -0.50 & 0.001 & -0.523 & 0.031 & -0.70 & 0.024 \\
\hline NSS & n.a. & n.a. & -0.12 & 0.479 & -0.06 & 0.812 & -0.69 & 0.028 \\
\hline Tibial NCV (m/s) & 0.41 & 0.236 & 0.34 & 0.042 & 0.60 & 0.007 & 0.67 & 0.034 \\
\hline Tibial CMAP $(\mu \mathrm{V})$ & 0.09 & 0.806 & 0.45 & 0.006 & 0.55 & 0.014 & 0.51 & 0.136 \\
\hline Tibial DML (ms) & -0.21 & 0.553 & 0.06 & 0.710 & -0.32 & 0.180 & -0.59 & 0.074 \\
\hline Peroneal NCV (m/s) & 0.53 & 0.117 & 0.41 & 0.014 & 0.70 & 0.001 & 0.85 & 0.003 \\
\hline Peroneal CMAP $(\mu \mathrm{V})$ & 0.15 & 0.675 & 0.58 & $<0.001$ & 0.64 & 0.003 & 0.64 & 0.066 \\
\hline Peroneal DML (ms) & -0.12 & 0.733 & 0.01 & 0.938 & -0.42 & 0.075 & -0.58 & 0.099 \\
\hline Sural NCV (m/s) & -0.06 & 0.877 & -0.03 & 0.887 & -0.15 & 0.597 & -0.05 & 0.927 \\
\hline Sural SNAP $(\mu V)$ & 0.38 & 0.279 & 0.32 & 0.069 & 0.34 & 0.167 & 0.26 & 0.540 \\
\hline $\operatorname{SAF}(A U)$ & -0.41 & 0.241 & -0.39 & 0.020 & -0.07 & 0.765 & -0.84 & 0.004 \\
\hline $\mathrm{PWV}(\mathrm{m} / \mathrm{s})$ & -0.70 & 0.037 & -0.45 & 0.004 & -0.51 & 0.027 & -0.59 & 0.094 \\
\hline IMT & -0.61 & 0.061 & -0.09 & 0.599 & -0.26 & 0.289 & -0.69 & 0.039 \\
\hline HbA1c (\%) & -0.47 & 0.242 & 0.13 & 0.422 & 0.05 & 0.859 & -0.35 & 0.329 \\
\hline Cystatin C (mg/l) & 0.41 & 0.317 & -0.37 & 0.038 & 0.07 & 0.800 & 0.31 & 0.420 \\
\hline Glomerular filtration rate $(\mathrm{ml} / \mathrm{min})$ & -0.37 & 0.369 & 0.45 & 0.012 & -0.17 & 0.512 & -0.26 & 0.506 \\
\hline Total serum cholesterol (mg/dl) & -0.12 & 0.770 & 0.26 & 0.109 & -0.20 & 0.423 & -0.30 & 0.397 \\
\hline HDL cholesterol (mg/dl) & -0.29 & 0.489 & 0.08 & 0.622 & -0.30 & 0.229 & -0.34 & 0.343 \\
\hline LDL cholesterol (mg/dl) & -0.03 & 0.937 & 0.24 & 0.149 & -0.11 & 0.687 & -0.24 & 0.502 \\
\hline Triglycerides (mg/dl) & -0.13 & 0.759 & 0.27 & 0.093 & -0.08 & 0.745 & 0.46 & 0.178 \\
\hline Troponin T (pg/dl) & -0.43 & 0.292 & -0.26 & 0.153 & 0.20 & 0.432 & -0.36 & 0.341 \\
\hline $\operatorname{proBNP}(\mathrm{pg} / \mathrm{dl})$ & -0.15 & 0.716 & -0.25 & 0.169 & 0.23 & 0.368 & 0.38 & 0.309 \\
\hline Years since smoke stop & n.a. & n.a. & n.a. & n.a. & -0.11 & 0.672 & n.a. & n.a. \\
\hline Cigarettes/day & n.a. & n.a. & n.a. & n.a. & n.a. & n.a. & -0.23 & 0.520 \\
\hline Years smoking & n.a. & n.a. & n.a. & n.a. & n.a. & n.a. & -0.37 & 0.365 \\
\hline Pack years & n.a. & n.a. & n.a. & n.a. & n.a. & n.a. & -0.32 & 0.445 \\
\hline
\end{tabular}

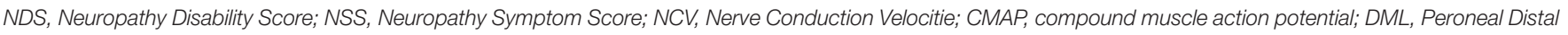

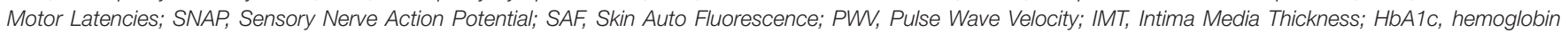

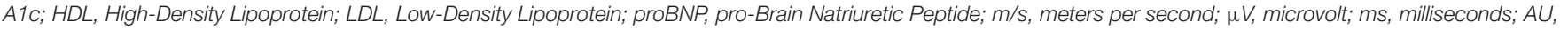
arbitrary units; $\mathrm{mg} / \mathrm{dl}$, milligram per deciliter; mg/l, milligram per liter; ml/min, milliliters per minute; pg/dl, picogram per deciliter. 
TABLE 3 | Correlation of the sciatic nerve's radial diffusivity (RD; in $10^{-5} \mathrm{~mm}^{2} / \mathrm{s}$ ) with demographic, clinical, apparatus-bound, and serological parameters.

\begin{tabular}{|c|c|c|c|c|c|c|c|c|}
\hline & \multicolumn{2}{|c|}{ RD controls } & \multicolumn{2}{|c|}{ RD T2D never-smokers } & \multicolumn{2}{|c|}{ RD T2D ex-smokers } & \multicolumn{2}{|c|}{ RD T2D smokers } \\
\hline & $r$ & $p$ & $r$ & $p$ & $r$ & $p$ & $r$ & $p$ \\
\hline Fractional anisotropy & -0.26 & 0.469 & -0.67 & $<0.001$ & -0.89 & $<0.001$ & -0.80 & 0.010 \\
\hline Axial diffusivity & 0.70 & 0.023 & 0.47 & 0.003 & 0.72 & 0.001 & 0.86 & 0.003 \\
\hline Sex & 0.11 & 0.760 & -0.07 & 0.655 & -0.24 & 0.307 & -0.17 & 0.655 \\
\hline Age (years) & -0.04 & 0.916 & 0.44 & 0.005 & 0.17 & 0.467 & 0.22 & 0.571 \\
\hline Body-mass index $\left(\mathrm{kg} / \mathrm{m}^{2}\right)$ & -0.27 & 0.456 & 0.06 & 0.732 & -0.09 & 0.696 & 0.68 & 0.042 \\
\hline NDS & -0.43 & 0.221 & 0.39 & 0.017 & 0.28 & 0.286 & 0.67 & 0.046 \\
\hline NSS & & & 0.26 & 0.114 & -0.22 & 0.403 & 0.44 & 0.242 \\
\hline Tibial NCV (m/s) & -0.33 & 0.358 & -0.26 & 0.125 & -0.50 & 0.028 & -0.48 & 0.186 \\
\hline Tibial CMAP $(\mu \mathrm{V})$ & $<0.01$ & 0.989 & -0.37 & 0.031 & -0.59 & 0.008 & -0.78 & 0.013 \\
\hline Tibial DML (ms) & 0.12 & 0.749 & -0.21 & 0.233 & 0.42 & 0.076 & 0.72 & 0.028 \\
\hline Peroneal NCV (m/s) & 0.14 & 0.696 & -0.34 & 0.046 & -0.49 & 0.034 & -0.86 & 0.006 \\
\hline Peroneal CMAP $(\mu \mathrm{V})$ & 0.27 & 0.456 & -0.51 & 0.002 & -0.56 & 0.012 & -0.83 & 0.012 \\
\hline Peroneal DML (ms) & 0.38 & 0.282 & -0.09 & 0.625 & 0.38 & 0.112 & 0.76 & 0.029 \\
\hline Sural NCV (m/s) & 0.35 & 0.322 & 0.10 & 0.612 & 0.31 & 0.266 & -0.26 & 0.670 \\
\hline Sural SNAP ( $\mu$ V) & 0.04 & 0.908 & -0.26 & 0.153 & -0.07 & 0.770 & 0.57 & 0.182 \\
\hline $\mathrm{SAF}(\mathrm{AU})$ & -0.19 & 0.591 & 0.44 & 0.009 & 0.07 & 0.765 & 0.59 & 0.112 \\
\hline $\mathrm{PWV}(\mathrm{m} / \mathrm{s})$ & -0.15 & 0.701 & 0.19 & 0.269 & 0.33 & 0.171 & 0.56 & 0.152 \\
\hline IMT (ratio) & 0.02 & 0.957 & 0.28 & 0.084 & 0.25 & 0.317 & 0.74 & 0.035 \\
\hline $\mathrm{HbA} 1 \mathrm{c}(\%)$ & -0.36 & 0.383 & -0.30 & 0.060 & -0.09 & 0.720 & 0.68 & 0.046 \\
\hline Cystatin C (mg/l) & 0.04 & 0.920 & 0.11 & 0.566 & -0.15 & 0.571 & 0.04 & 0.932 \\
\hline Glomerular filtration rate (ml/min) & $<0.01$ & 0.994 & -0.13 & 0.499 & 0.21 & 0.428 & -0.01 & 0.988 \\
\hline Total serum cholesterol (mg/dl) & 0.36 & 0.384 & -0.29 & 0.073 & -0.06 & 0.802 & 0.63 & 0.068 \\
\hline HDL cholesterol (mg/dl) & -0.11 & 0.792 & -0.05 & 0.757 & 0.14 & 0.593 & 0.43 & 0.243 \\
\hline LDL cholesterol (mg/dl) & 0.42 & 0.295 & -0.21 & 0.216 & -0.11 & 0.666 & 0.44 & 0.234 \\
\hline Triglycerides (mg/dl) & 0.18 & 0.670 & -0.33 & 0.043 & -0.01 & 0.964 & 0.20 & 0.608 \\
\hline Troponin T (pg/dl) & -0.55 & 0.155 & 0.30 & 0.108 & -0.29 & 0.241 & 0.71 & 0.040 \\
\hline proBNP (pg/dl) & 0.06 & 0.879 & 0.26 & 0.166 & -0.27 & 0.276 & -0.46 & 0.257 \\
\hline Years since smoke stop & n.a. & n.a. & n.a. & n.a. & 0.03 & 0.902 & n.a. & n.a. \\
\hline Cigarettes/day & n.a. & n.a. & n.a. & n.a. & n.a. & n.a. & 0.31 & 0.422 \\
\hline Years smoking & n.a. & n.a. & n.a. & n.a. & n.a. & n.a. & -0.21 & 0.651 \\
\hline Pack years & n.a. & n.a. & n.a. & n.a. & n.a. & n.a. & 0.03 & 0.946 \\
\hline
\end{tabular}

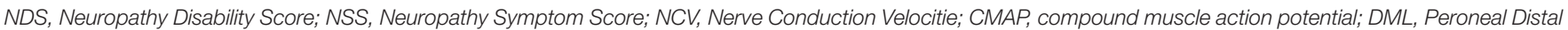

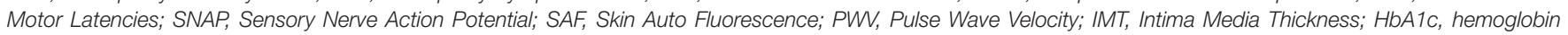

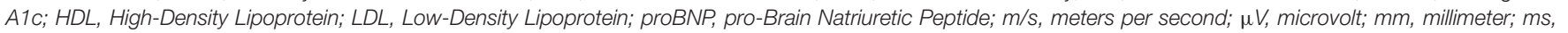
milliseconds; $A U$, arbitrary units; mg/dl, milligram per deciliter; mg/l, milligram per liter; ml/min, milliiters per minute; pg/dl, picogram per deciliter.

RD showed correlations with peroneal NCV and CMAP in never-smokers $(r=-0.34 ; p=0.046$ and $r=-0.51$; $p=0.002)$, ex-smokers $(r=-0.49 ; p=0.034$ and $r=-0.56 ; p=0.012)$ and smokers $(r=-0.86 ; p=0.033$ and $r=-0.83 ; p=0.012$ ) as well as correlations with tibial CMAP in never smokers $(r=-0.37 ; p=0.031)$, ex-smokers $(r=-0.59$; $p=0.008)$ and smokers $(r=-0.78 ; p=0.013)$ with T2D.

$\mathrm{AD}$ was correlated with tibial DML in ex-smokers and smokers ( $r=0.51 ; p=0.032$ and $r=0.64 ; p=0.046$, respectively) with T2D. In smokers with T2D, AD was further correlated with peroneal NCV $(r=-0.73 ; p=0.027)$ and peroneal CMAP $(r=-0.85 ; p=0.004)$.

\section{Correlation With Additional Paraclinical Data}

FA showed negative correlations with the pulse wave velocity in controls $(r=-0.70 ; p=0.037)$, never-smokers $(r=-0.45$; $p=0.004)$, ex-smokers $(r=-0.51 ; p=0.027)$, and a similar trend in smokers $(r=-0.59 ; p=0.094)$ with T2D. In smokers with T2D, IMT as a surrogate marker of cardiovascular disease in diabetes (Sibal et al., 2011) was negatively correlated with FA $(r=-0.69$; $p=0.039)$. Negative correlations were found between FA and SAF, a measure of the tissue deposition of advanced glycation end product and therefore of the degree of glycemic stress in diabetes, in never-smokers $(r=-0.39 ; p=0.020)$ and smokers $(r=-0.84$; $p=0.004)$, but not in ex-smokers with T2D $(r=-0.07 ; p=0.765)$.

$\mathrm{RD}$ was positively correlated with the IMT in smokers $(r=0.74 ; p=0.035)$. No correlations were found between RD and PWV. RD was positively correlated with skin auto-fluorescence in never-smokers $(r=0.44 ; p=0.009)$ and a similar trend was seen in smokers $(r=0.59 ; p=0.122)$ but not in ex-smokers $(r=0.07$; $p=0.765)$. 
TABLE 4 | Correlation of the sciatic nerve's axial diffusivity (AD; in $10^{-5} \mathrm{~mm}^{2} / \mathrm{s}$ ) with demographic, clinical, apparatus-bound, and serological parameters.

\begin{tabular}{|c|c|c|c|c|c|c|c|c|}
\hline & \multicolumn{2}{|c|}{ AD controls } & \multicolumn{2}{|c|}{ AD T2D never-smokers } & \multicolumn{2}{|c|}{ AD T2D ex-smokers } & \multicolumn{2}{|c|}{ AD T2D smokers } \\
\hline & $r$ & $p$ & $r$ & $p$ & $r$ & $p$ & $r$ & $p$ \\
\hline Fractional anisotropy & 0.36 & 0.305 & 0.29 & 0.071 & -0.35 & 0.144 & -0.65 & 0.044 \\
\hline Radial diffusivity & 0.70 & 0.023 & 0.47 & 0.003 & 0.72 & 0.001 & 0.86 & 0.003 \\
\hline Sex & 0.36 & 0.306 & 0.02 & 0.918 & -0.07 & 0.767 & 0.13 & 0.727 \\
\hline Age (years) & -0.48 & 0.166 & -0.01 & 0.936 & -0.01 & 0.965 & 0.13 & 0.721 \\
\hline Body-mass index $\left(\mathrm{kg} / \mathrm{m}^{2}\right)$ & -0.45 & 0.193 & -0.32 & 0.048 & -0.23 & 0.342 & 0.76 & 0.011 \\
\hline NDS & -0.42 & 0.225 & -0.05 & 0.779 & -0.18 & 0.493 & 0.44 & 0.207 \\
\hline NSS & & & 0.26 & 0.106 & -0.48 & 0.049 & 0.44 & 0.199 \\
\hline Tibial NCV (m/s) & -0.09 & 0.806 & 0.02 & 0.906 & -0.17 & 0.511 & -0.26 & 0.466 \\
\hline Tibial CMAP $(\mu \mathrm{V})$ & 0.04 & 0.916 & 0.09 & 0.595 & -0.41 & 0.088 & -0.61 & 0.062 \\
\hline Tibial DML (ms) & 0.11 & 0.760 & -0.18 & 0.305 & 0.51 & 0.032 & 0.64 & 0.046 \\
\hline Peroneal NCV (m/s) & 0.36 & 0.313 & 0.04 & 0.808 & 0.08 & 0.753 & -0.73 & 0.027 \\
\hline Peroneal CMAP $(\mu \mathrm{V})$ & 0.42 & 0.225 & 0.06 & 0.736 & -0.07 & 0.776 & -0.85 & 0.004 \\
\hline Peroneal DML (ms) & 0.32 & 0.366 & -0.10 & 0.573 & 0.08 & 0.763 & 0.66 & 0.053 \\
\hline Sural NCV (m/s) & 0.54 & 0.110 & 0.02 & 0.939 & 0.29 & 0.311 & 0.17 & 0.750 \\
\hline Sural SNAP $(\mu V)$ & 0.56 & 0.090 & 0.08 & 0.676 & 0.39 & 0.120 & 0.09 & 0.834 \\
\hline $\mathrm{SAF}(\mathrm{AU})$ & -0.55 & 0.101 & 0.22 & 0.205 & -0.07 & 0.786 & 0.57 & 0.109 \\
\hline $\mathrm{PWV}(\mathrm{m} / \mathrm{s})$ & -0.45 & 0.225 & -0.19 & 0.264 & $<0.01$ & 0.985 & 0.30 & 0.438 \\
\hline IMT & -0.22 & 0.548 & 0.21 & 0.201 & -0.04 & 0.882 & 0.62 & 0.077 \\
\hline HbA1c (\%) & -0.22 & 0.605 & -0.28 & 0.076 & -0.13 & 0.630 & 0.69 & 0.027 \\
\hline Cystatin C (mg/l) & 0.44 & 0.271 & -0.31 & 0.086 & -0.19 & 0.485 & -0.05 & 0.900 \\
\hline Glomerular filtration rate $(\mathrm{ml} / \mathrm{min})$ & -0.38 & 0.355 & 0.38 & 0.040 & 0.20 & 0.466 & 0.21 & 0.594 \\
\hline Total serum cholesterol (mg/dl) & 0.38 & 0.355 & -0.02 & 0.920 & -0.30 & 0.246 & 0.48 & 0.157 \\
\hline HDL cholesterol (mg/dl) & 0.03 & 0.938 & -0.03 & 0.872 & -0.06 & 0.830 & 0.10 & 0.789 \\
\hline LDL cholesterol (mg/dl) & 0.38 & 0.359 & 0.14 & 0.417 & -0.28 & 0.289 & 0.44 & 0.201 \\
\hline Triglycerides (mg/dl) & 0.30 & 0.468 & -0.11 & 0.518 & -0.12 & 0.658 & 0.24 & 0.512 \\
\hline Troponin T (pg/dl) & -0.44 & 0.271 & 0.06 & 0.769 & -0.34 & 0.180 & 0.42 & 0.255 \\
\hline $\operatorname{proBNP}(\mathrm{pg} / \mathrm{dl})$ & -0.01 & 0.976 & 0.01 & 0.974 & -0.30 & 0.248 & -0.58 & 0.105 \\
\hline Years since smoke stop & n.a. & n.a. & n.a. & n.a. & -0.14 & 0.590 & n.a. & n.a. \\
\hline Cigarettes/day & n.a. & n.a. & n.a. & n.a. & n.a. & n.a. & 0.41 & 0.245 \\
\hline Years smoking & n.a. & n.a. & n.a. & n.a. & n.a. & n.a. & -0.22 & 0.600 \\
\hline Pack years & n.a. & n.a. & n.a. & n.a. & n.a. & n.a. & -0.05 & 0.914 \\
\hline
\end{tabular}

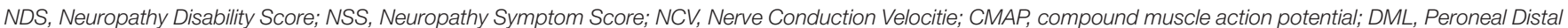

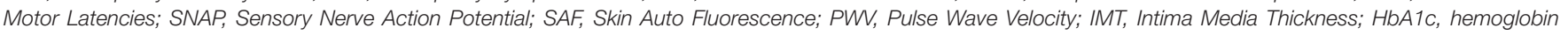

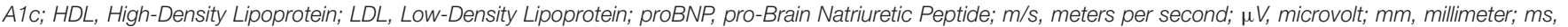
milliseconds; $A U$, arbitrary units; mg/l, milligram per liter; ml/min, milliliters per minute; mg/dl, milligram per deciliter; pg/dl, picogram per deciliter.

\section{Correlation With Serologic Data}

In never-smokers, FA showed negative correlations with cystatin $\mathrm{c}$ and positive correlations with GFR $(r=-0.37 ; p=0.038$ and $r=0.45 ; p=0.012$ ). No correlations were found between FA and HbAlc levels, cholesterol levels or triglycerides.

In smokers, $\mathrm{RD}$ was correlated with HbAlc levels $(r=0.68$; $p=0.046)$ and hsTNT $(r=0.71 ; p=0.048)$. No further correlations of $\mathrm{AD}$ with serologic parameters were found in any of the groups. A detailed summary of all correlations of imaging parameters is given in Tables 2-4.

\section{DISCUSSION}

The key findings of this study were that (i) the sciatic nerve's FA is lower in smokers and ex-smokers with T2D compared to agematched controls, and that the FA in smokers with T2D is lower than in never-smokers with T2D; (ii) in all T2D patient groups, FA was negatively correlated with the NDS, tibial and peroneal
NCV and CMAP, and PWV; (iii) in never-smokers and smokers, FA was negatively correlated with SAF, whereas in ex-smokers no such correlation could be found; and (iv) in smokers, RD was positively correlated with hsTNT, SAF, BMI, and HbAlc levels.

The finding that the sciatic nerve's FA was lower in smokers compared to controls and never-smokers suggests that cigarette smoking contributes to nerve damage in patients with T2D, and that, therefore, to quit smoking may pose a useful preventive measure to slow the progression of DPN, as suggested by previous studies (Kar et al., 2016). The finding of a lower FA in ex-smokers compared to controls suggests that the negative effect of smoking on structural nerve integrity in T2D is not completely reversible once a patient quits smoking, supporting previous studies that found smoking prevention in adolescents with diabetes to be of importance with regards to the development and progression of DPN (Jaiswal et al., 2017; Christensen et al., 2020). The finding that $\mathrm{RD}$, but not $\mathrm{AD}$ was higher in smokers with $\mathrm{T} 2 \mathrm{D}$ when compared to controls and never-smokers with $\mathrm{T} 2 \mathrm{D}$, suggests that smoking primarily causes damage to the myelin sheath in T2D, 
since RD has previously been shown to be a marker for myelin integrity (Heckel et al., 2015; Vaeggemose et al., 2017; Kurz et al., 2018). The correlations of FA and RD with electrophysiological parameters and clinical scores indicate that both parameters pose reliable parameters for structural nerve integrity in T2D as suggested by recent studies (Jende et al., 2021).

The finding that FA was negatively correlated with SAF in smokers, but not in ex-smokers, suggests that the deposition of advanced glycation end products in or around peripheral nerves and subsequent myelin damage may be more relevant in active smokers than in ex-smokers. In contrast, the absence of a correlation between FA and SAF in ex-smokers and the findings that both hsTNT and PWV, markers of microangiopathy in T2D (Jende et al., 2020a), are higher in ex-smokers versus controls, while PWV was not significantly associated with FA decrease in smokers, may suggest that microvascular impairment associated nerve damage predominates over effects of hyperglycemia in T2D ex-smokers.

The correlation of RD with IMT, hsTNT and HbA1c levels in smokers further supports the hypothesis that hyperglycemia, macroangiopathy, and microangiopathy induced by smoking contribute to myelin damage in T2D in the sense of a "triple hit" (Nilsson et al., 2004; Christensen et al., 2020). The finding that the sciatic nerve's RD was positively correlated with the BMI in smokers further supports the hypothesis that the impact of smoking on nerve integrity is worse in obese patients with T2D (Jaiswal et al., 2017).

The results of this study are of importance with regard to the effect of glucose control on the progression of DPN in T2D: since former studies have found that normalization of blood glucose levels in T2D is not beneficial for slowing the progression of DPN, it might be of interest to future studies to assess whether the effect of glucose control in T2D differs between smokers, ex-smokers and never-smokers.

This explorative study is limited by the fact that only crosssectional data were analyzed, which does not allow for causative conclusions on the effects of smoking on myelin damage in T2D. Another limitation is the fact that our sample size, in particular the number of active smokers, is too small for multivariate analysis of all potential confounders. One must consider, however, that all patient groups were matched for gender, age, BMI, HbA1c levels, parameters of renal function, and total serum cholesterol. It is therefore unlikely that the effects found in our cohort are caused by other effects than those induced by cigarette smoking.

One may of course argue that we did not separate DPN patients from patients without DPN. However, it should be noted that all patient groups were matched for NDS and that previous MRN imaging studies have found that DPN is a continuous process during which nerve lesions accumulate in asymptomatic patients who start to experience symptoms once a certain amount of structural nerve damage is reached (Groener et al., 2019). Therefore, assessing the sciatic nerve's structural integrity with MRN imaging parameters allows for more accurate correlations and group comparisons than dividing patients into two groups based on clinical scores (Vaeggemose et al., 2017; Jende et al., 2020a).
In summary, this study is the first to find that structural nerve integrity assessed by $\mathrm{MR}$ neurography is lower in smokers with T2D compared to ex-smokers with T2D and age-matched controls, which is most likely due to damage to the affected nerves' myelin sheath. Our findings suggest that in smokers with T2D, hyperglycemia, obesity, micro- and macroangiopathy contribute to myelin damage, whereas in exsmokers microangiopathy appears to be the main contributing factor. Our results indicate that cigarette smoking is harmful to peripheral nerves in $\mathrm{T} 2 \mathrm{D}$ and that, therefore, interventions to stop smoking may be promising preventive measures to slow down the progression of DPN. Further longitudinal studies are required to verify this hypothesis.

\section{DATA AVAILABILITY STATEMENT}

The datasets presented in this article are not readily available because they contain sensitive patient information. The data supporting the conclusions of this article will be made available upon reasonable request by any qualified researcher. Requests to access the datasets should be directed to FK, felix.kurz@med. uni-heidelberg.de.

\section{ETHICS STATEMENT}

The studies involving human participants were reviewed and approved by Ethikkommission der medizinischen Fakultät der Universität Heidelberg, Alte Glockengießerei 11/1, 69115 Heidelberg, Germany. The patients/participants provided their written informed consent to participate in this study.

\section{AUTHOR CONTRIBUTIONS}

JJ, MB, SH, PN, and FK designed and coordinated the study. JJ, CM, JG, AJ, and FK contributed to the organization of participants. JJ, AJ, and FK collected MR data. FK developed image analysis tools. ZK, JG, and SK collected clinical, serological, and electrophysiological data. JJ and FK analyzed the data and wrote the manuscript with input from all coauthors. All authors contributed to the article and approved the submitted version.

\section{FUNDING}

This study was supported by the German Research Foundation (SFB 1158, 1118) and the International Foundation for Research in Paraplegia (IRP). Both DFG and IRP had no influence on the study design, collection, and analysis of data or on the writing of the article.

\section{ACKNOWLEDGMENTS}

We thank Dorothea Willich (Department of Neuroradiology, Heidelberg University Hospital) for her ongoing support and excellent technical performance of all MRN examinations. 


\section{REFERENCES}

Alleman, C. J. M., Westerhout, K. Y., Hensen, M., Chambers, C., Stoker, M., Long, S., et al. (2015). Humanistic and economic burden of painful diabetic peripheral neuropathy in Europe: a review of the literature. Diabet. Res. Clin. Pract. 109, 215-225. doi: 10.1016/j.diabres.2015.04.031

Benbow, S. J., Williams, G., and MacFarlane, I. A. (1997). Smoking habits and painful diabetic neuropathy. J. Diabet. Complications 11, 334-337.

Christensen, D. H., Knudsen, S. T., Gylfadottir, S. S., Christensen, L. B., Nielsen, J. S., Beck-Nielsen, H., et al. (2020). Metabolic factors, lifestyle habits, and possible polyneuropathy in early type 2 diabetes: a nationwide study of 5,249 patients in the danish centre for strategic research in type 2 diabetes (DD2) cohort. Diabet. Care 43, 1266-1275. doi: 10.2337/dc19-2277

Christidi, F., Karavasilis, E., Samiotis, K., Bisdas, S., and Papanikolaou, N. (2016). Fiber tracking: a qualitative and quantitative comparison between four different software tools on the reconstruction of major white matter tracts. Eur. J. Radiol. Open 3, 153-161. doi: 10.1016/j.ejro.2016.06.002

Clair, C., Cohen, M. J., Eichler, F., Selby, K. J., and Rigotti, N. A. (2015). The effect of cigarette smoking on diabetic peripheral neuropathy: a systematic review and meta-analysis. J. Gen. Intern. Med. 30, 1193-1203. doi: 10.1007/s11606-0153354-y

Corine van de Zande, S., de Vries, J. K., Akker-Scheek, I., van den Zwerver, J., and Smit, A. J. (2020). A physically active lifestyle is related to a lower level of skin autofluorescence in a large population with chronic-disease (LifeLines cohort). J. Sport Health Sci. S2095-2546(20)30123-X. doi: 10.1016/J.JSHS.2020.09.007

Csordas, A., and Bernhard, D. (2013). The biology behind the atherothrombotic effects of cigarette smoke. Nat. Publ. Group 10, 219-230. doi: 10.1038/nrcardio. 2013.8

Elliott, J., Tesfaye, S., Chaturvedi, N., Gandhi, R. A., Stevens, L. K., Emery, C., et al. (2009). Large-fiber dysfunction in diabetic peripheral neuropathy is predicted by cardiovascular risk factors. Diabet. Care 32, 1896-1900. doi: 10.2337/dc090554

Fernando, M. E., Crowther, R. G., Lazzarini, P. A., Sangla, K. S., Wearing, S., Buttner, P., et al. (2019). Within- and between-body-site agreement of skin autofluorescence measurements in people with and without diabetesrelated foot disease. J. Diabet. Sci. Technol. 13, 836-846. doi: 10.1177/ 1932296819853555

Gaede, P., Vedel, P., Parving, H.-H. H., Pedersen, O., Aagenaes, O., and Parving, H.-H. H. (1999). Intensified multifactorial intervention in patients with type 2 diabetes mellitus and microalbuminuria: the Steno type 2 randomised study. Lancet 353, 617-622. doi: 10.1016/S0140-6736(98)07368-1

Groener, J. B., Jende, J. M. E., Kurz, F. T., Kender, Z., Treede, R.-D., Schuh-Hofer, S., et al. (2019). Understanding diabetic neuropathy: from subclinical nerve lesions to severe nerve fiber deficits. A cross-sectional study in patients with type 2 diabetes and healthy controls. Diabetes 69, 436-447. doi: 10.2337/db190197

Heckel, A., Weiler, M., Xia, A., Ruetters, M., Pham, M., Bendszus, M., et al. (2015). Peripheral nerve diffusion tensor imaging: assessment of axon and myelin sheath integrity. PLoS One 10:e0130833. doi: 10.1371/journal.pone.013 0833

Jaiswal, M., Divers, J., Dabelea, D., Isom, S., Bell, R. A., Martin, C. L., et al. (2017). Prevalence of and risk factors for diabetic peripheral neuropathy in youth with type 1 and type 2 diabetes: search for diabetes in youth study. Diabet. Care 40, 1226-1232. doi: $10.2337 / \mathrm{dc} 17-0179$

Jende, J. M. E., Kender, Z., Rother, C., Alvarez-Ramos, L., Groener, J. B., Pham, M., et al. (2020c). Diabetic polyneuropathy is associated with pathomorphological changes in human dorsal root ganglia: a study using 3T MR neurography. Front. Neurosci. 14:570744. doi: 10.3389/fnins.2020.570744

Jende, J. M. E., Groener, J. B., Kender, Z., Rother, C., Hahn, A., Hilgenfeld, T., et al. (2020b). Structural nerve remodeling at 3-T MR neurography differs between painful and painless diabetic polyneuropathy in type 1 or 2 diabetes. Radiology 294, 405-414. doi: 10.1148/radiol.201919 1347

Jende, J. M. E., Groener, J. B., Kender, Z., Hahn, A., Morgenstern, J., Heiland, S., et al. (2020a). Troponin T parallels structural nerve damage in type 2 diabetes: a cross-sectional study using magnetic resonance neurography. Diabetes 69, 713-723. doi: $10.2337 / \mathrm{db} 19-1094$
Jende, J. M. E., Kender, Z., Mooshage, C., Groener, J. B., Alvarez-Ramos, L., Kollmer, J., et al. (2021). Diffusion tensor imaging of the sciatic nerve as a surrogate marker for nerve functionality of the upper and lower limb in patients with diabetes and prediabetes. Front. Neurosci. 15:642589. doi: 10.3389/fnins. 2021.642589

Kar, D., Gillies, C., Zaccardi, F., Webb, D., Seidu, S., Tesfaye, S., et al. (2016). Relationship of cardiometabolic parameters in non-smokers, current smokers, and quitters in diabetes: a systematic review and metaanalysis. Cardiovasc. Diabetol. 15, 158-158. doi: 10.1186/s12933-016-04 75-5

Kim, H.-L., and Kim, S.-H. (2019). Pulse wave velocity in atherosclerosis. Front. Cardiovasc. Med. 6:41. doi: 10.3389/fcrm.2019.00041

Kurz, F. T., Buschle, L. R., Hahn, A., Jende, J. M. E., Bendszus, M., Heiland, S., et al. (2018). Diffusion effects in myelin sheath free induction decay. J. Magn. Reson. $297,61-75$.

Levey, A. S., Stevens, L. A., Schmid, C. H., Zhang, Y. L., Castro, A. F., Feldman, H. I., et al. (2009). A new equation to estimate glomerular filtration rate. Ann. Intern. Med. 150, 604-612.

Natus Medical Incorporated (2020). Nicolet VikingQuest User Guide Version 21.1. Pleasanton, CA: Natus Medical Incorporated.

Nilsson, P. M., Gudbjörnsdottir, S., Eliasson, B., Cederholm, J., and Steering Committee of the Swedish National Diabetes Register. (2004). Smoking is associated with increased $\mathrm{HbAlc}$ values and microalbuminuria in patients with diabetes-data from the national diabetes register in Sweden. Diabet. Metab. 30, 261-268.

Shillo, P., Sloan, G., Greig, M., Hunt, L., Selvarajah, D., Elliott, J., et al. (2019). Painful and painless diabetic neuropathies: what is the difference? Curr. Diabet. Rep. 19:32. doi: 10.1007/s11892-019-1150-5

Sibal, L., Agarwal, S. C., and Home, P. D. (2011). Carotid intima-media thickness as a surrogate marker of cardiovascular disease in diabetes. Diabet. Metab. Syndr. Obes. 4, 23-34. doi: 10.2147/DMSO.S8540

Stålberg, E., van Dijk, H., Falck, B., Kimura, J., Neuwirth, C., Pitt, M., et al. (2019). Standards for quantification of EMG and neurography. Clin. Neurophysiol. 130, 1688-1729. doi: 10.1016/j.clinph.2019.05.008

Tesfaye, S., Chaturvedi, N., Eaton, S. E. M., Ward, J. D., Manes, C., IonescuTirgoviste, C., et al. (2005). Vascular risk factors and diabetic neuropathy. N. Engl. J. Med. 352, 341-350. doi: 10.1056/NEJMoa032782

Toth, P. P., Simko, R. J., Palli, S. R., Koselleck, D., Quimbo, R. A., and Cziraky, M. J. (2012). The impact of serum lipids on risk for microangiopathy in patients with type 2 diabetes mellitus. Cardiovasc. Diabetol. 11:109. doi: 10.1186/1475-284011-109

Vaeggemose, M., Pham, M., Ringgaard, S., Tankisi, H., Ejskjaer, N., Heiland, S., et al. (2017). Diffusion tensor imaging MR neurography for the detection of polyneuropathy in type 1 diabetes. J. Magn. Reson. Imaging 45, 1125-1134. doi: $10.1002 /$ imri.25415

Young, M. J., Boulton, A. J., MacLeod, A. F., Williams, D. R., and Sonksen, P. H. (1993). A multicentre study of the prevalence of diabetic peripheral neuropathy in the United Kingdom hospital clinic population. Diabetologia 36, 150-154. doi: 10.1007/BF00400697

Conflict of Interest: The authors declare that the research was conducted in the absence of any commercial or financial relationships that could be construed as a potential conflict of interest.

Publisher's Note: All claims expressed in this article are solely those of the authors and do not necessarily represent those of their affiliated organizations, or those of the publisher, the editors and the reviewers. Any product that may be evaluated in this article, or claim that may be made by its manufacturer, is not guaranteed or endorsed by the publisher.

Copyright (C) 2022 Jende, Mooshage, Kender, Kopf, Groener, Heiland, Juerchott, Nawroth, Bendszus and Kurz. This is an open-access article distributed under the terms of the Creative Commons Attribution License (CC BY). The use, distribution or reproduction in other forums is permitted, provided the original author(s) and the copyright owner(s) are credited and that the original publication in this journal is cited, in accordance with accepted academic practice. No use, distribution or reproduction is permitted which does not comply with these terms. 